\title{
Towards a molecular classification of colorectal cancer
}

\author{
Alessandro Lugli * \\ Clinical Pathology Division, Institute of Pathology, University of Bern, Bern, Switzerland \\ ${ }^{*}$ Correspondence: alessandro.lugli@pathology.unibe.ch \\ Edited by: \\ Mark De Ridder, Vrije Universiteit Brussel, Belgium \\ Reviewed by: \\ Jean-Pierre Gérard, Université de Nice, France
}

Keywords: colorectal cancer, molecular classification, Cdx2, Wnt signaling pathway, MGMT, telomere length, Ras signaling pathway, microRNA

In colorectal cancer (CRC), an internationally standardized molecular classification has not been implemented yet. Nevertheless, there are different pathogenetic aspects that could form the basis for a future molecular classification in CRC (1).

In the classic model, CRC is divided into two major pathways. The first is the chromosomal instability pathway including $85 \%$ of all CRCs. This pathway is based on the adenoma-carcinoma sequence, which is defined by consecutive mutations in the APC, KRAS, and TP53 gene and deletion of the chromosome $18 \mathrm{q}$ (2). An example of an autosomal dominantly inherited disease, which belongs to the chromosomal instability pathway group, is the familial adenomatous polyposis syndrome (FAP). The second, namely the microsatellite instability pathway $(15 \%$ of all CRCs) is defined by a mismatch-repair deficiency, which leads to a genomic instability. The microsatellite instability status can be sporadic $(12 \%)$ or hereditary (3\%, Lynch syndrome-associated CRC, HNPCC). Sporadic cases can be differentiated from hereditary cases by using the Amsterdam/Revised Bethesda criteria.

In 2007, Jeremy Jass proposed a molecular classification based on clinical, morphological, and molecular parameters, which included five subgroups (3).

Group 1 (12\% of all CRCs): chromosomally stable, MLH1 methylated, MSI-H, BRAF mutated, CpG island methylator phenotype (CIMP) high status; group 2 ( $8 \%$ of all CRCs): chromosomally stable, partially MLH1 methylated, microsatellite stable (MSS, MSI-L), BRAF mutated, CIMP high; group 3 (20\% of all CRCs): chromosomally instable, MGMT methylated, MSS/MSI-L, KRAS mutated, CIMP low; group 4 (57\% of all CRCs): chromosomally instable, MSS/MSI-L, CIMP negative; group 5 (3\% of all CRCs): chromosomally stable, MSI-H, CIMP negative, BRAF wild type.

Following up on this last classification, in 2010, Barbara Leggett and Vicki Whitehall published a pathogenetic overview of sporadic CRC including three pathways (4).

The serrated pathway: MSI-H, CIMP high, BRAF mutated (corresponds to the Jass group 1); the alternative pathway: MSS, CIMP low, KRAS mutated (corresponds to the Jass group 3; the traditional pathway: MSS, CIMP negative, BRAT wild type, KRAS wild type (corresponds to the Jass group 4).

In 2012, the Cancer Genome Atlas Network published a promising approach for a future molecular classification based on different pathways (5).

Group 1 (wnt and TGF-beta pathway): proliferation, stem cell, and progenitor phenotype; group 2 (PIK3CA and RTK-RAS pathway): proliferation, cell survival, translation; group 3 (p53 pathway): proliferation, cell survival. This approach may elucidate promising target molecules for wnt pathway inhibitors or proteins of the PIK3CA and RTK-RAS pathway such as IGF2, IGFR, ERBB2, ERBB3, MEK, AKT, and MTOR.

Recently, a new proposal for a molecular classification of CRC was published by a research group from Oxford based on 906 stage II and III CRCs (6).

Group 1: MSI-H and/or BRAF mutated; group 2: chromosomally instable and/or TP53 mutated with KRAS and PIK3Ca wild type status; group 3: chromosomally instable, KRAS and/or PIK3CA mutated; TP53 wild type status; group 4: chromosomally stable, KRAS and/or PIK3CA mutated; TP53 wild type status; group 5: NRAS mutated; group 6: no mutations; group 7: other.

All these proposals for a CRC molecular classification have in common that some molecular features such as KRAS, BRAF, microsatellite status, and CIMP are often included. Nevertheless, it has to be kept in mind that molecular markers can be prognostic (i.e., BRAF), predictive (i.e., RAS), or both (i.e., microsatellite status). Therefore, the main aim of the research topic "Toward a molecular classification of colorectal cancer" was to include articles that focus on the role of already established (7-13) or potentially novel and promising molecular biomarkers such as telomere length (14) or microRNAs (15) and additionally to give an overview on the molecular pathology of CRC.

\section{REFERENCES}

1. Bosman FT, Carneiro F, et al. WHO Classification of Tumours of the Digestive System. 3rd ed. Lyon: IARC (2010). p. 131-82.

2. Fearon ER, Vogelstein B. A genetic model for colorectal tumorigenesis. Cell (1990) 61(5):759-67. doi:10.1016/0092-8674(90)90186-I

3. Jass JR. Classification of colorectal cancer based on correlation of clinical, morphological and molecular features. Histopathology (2007) 50(1):113-30. doi:10.1111/j.1365-2559.2006.02549.x

4. Leggett B, Whitehall V. Role of the serrated pathway in colorectal cancer pathogenesis. Gastroenterology (2010) 138(6):2088-100. doi:10.1053/j.gastro. 2009.12.066

5. The Cancer Genome Atlas Network. Comprehensive molecular characterization of human colon and rectal cancer. Nature (2012) 487(7407):330-7. doi:10.1038/nature11252

6. Domingo E, Ramamoorthy R, Oukrif D, Rosmarin D, Presz M, Wang H, et al. Use of multivariate analysis to suggest a new molecular classification of colorectal cancer. J Pathol (2013) 229(3):441-8. doi:10.1002/path.4139

7. Dawson H, Koelzer VH, Lukesch AC, Mallaev M, Inderbitzin D, Lugli A, et al. Loss of Cdx2 expression in primary tumors and lymph node metastases is specific for mismatch repair-deficiency in colorectal cancer. Front Oncol (2013) 3:265. doi:10.3389/fonc.2013.00265

8. Minoo P. Toward a molecular classification of colorectal cancer: the role of MGMT. Front Oncol (2013) 3:266. doi:10.3389/fonc.2013.00266

9. Heinimann K. Toward a molecular classification of colorectal cancer: the role of microsatellite instability status. Front Oncol (2013) 3:272. doi:10.3389/fonc. 2013.00272 
10. Thiel A, Ristimäki A. Toward a molecular classification of colorectal cancer: the role of BRAF. Front Oncol (2013) 3:281. doi:10.3389/fonc.2013.00281

11. Molinari F, Frattini M. Functions and regulation of the PTEN gene in colorectal cancer. Front Oncol (2014) 3:326. doi:10.3389/fonc.2013.00326

12. Cathomas G. PIK3CA in colorectal cancer. Front Oncol (2014) 4:35. doi:10.3389/ fonc.2014.00035

13. Bruun J, Kolberg M, Nesland JM, Svindland A, Nesbakken A, Lothe RA. Prognostic significance of $\beta$-catenin, E-cadherin, and SOX9 in colorectal cancer: results from a large population-representative series. Front Oncol (2014) 4:118. doi:10.3389/fonc.2014.00118

14. Baichoo E, Boardman LA. Toward a molecular classification of colorectal cancer: the role of telomere length. Front Oncol (2014) 4:158. doi:10.3389/fonc.2014. 00158

15. Khatri R, Subramanian S. MicroRNA-135b and its circuitry networks as potential therapeutic targets in colon cancer. Front Oncol (2013) 3:268. doi:10.3389/ fonc. 2013.00268
Conflict of Interest Statement: The author declares that the research was conducted in the absence of any commercial or financial relationships that could be construed as a potential conflict of interest.

Received: 16 October 2014; accepted: 10 February 2015; published online: 25 February 2015.

Citation: Lugli A (2015) Towards a molecular classification of colorectal cancer. Front. Oncol. 5:46. doi: 10.3389/fonc.2015.00046

This article was submitted to Gastrointestinal Cancers, a section of the journal Frontiers in Oncology.

Copyright $\odot 2015$ Lugli. This is an open-access article distributed under the terms of the Creative Commons Attribution License (CC BY). The use, distribution or reproduction in other forums is permitted, provided the original author(s) or licensor are credited and that the original publication in this journal is cited, in accordance with accepted academic practice. No use, distribution or reproduction is permitted which does not comply with these terms. 